\title{
ANÁLISE CRÍTICA DA ESCOLHA DE PORTFÓLIO NO MERCADO DE CAPITAIS BRASILEIRO COM APLICAÇÃO DE MODELAGEM EM PROGRAMAÇÃO INTEIRA E RESTRIÇÕES DE BENJAMIN GRAHAM
}

\author{
Octavio Sanz dos Santos Thomé ${ }^{1}$ \\ Ricardo Bordeaux-Rêgo ${ }^{2}$
}

\begin{abstract}
Resumo: Este trabalho objetiva analisar uma ferramenta de auxílio à tomada de decisão na escolha de uma carteira de ações no mercado de capitais brasileiro. Tal ferramenta conta com os fundamentos consagrados por Benjamin Graham, uma referência para investidores desde a década de trinta do século passado. Nesse sentido, alguns dos parâmetros utilizados por Graham para investimento em ações são modelados em programação inteira através do VBA (Excel), cuja solução é obtida utilizando a biblioteca de funções UFFLP. Foram propostas duas modelagens: a primeira minimizando o índice P/L, e a segunda, maximizando dividendos. Das carteiras resultantes destaca-se para a participação significativa de empresas dos setores de construção civil e energia elétrica, sendo as primeiras em função dos baixos índices preço sobre lucro e as últimas em função dos elevados dividendos. Os resultados apontam que o modelo de Graham não se aplicaria aos ativos avaliados, talvez devido a simplificações no seu emprego.
\end{abstract}

Palavras-chave: Mercado de Capitais, Programação Inteira, Benjamin Graham.

\begin{abstract}
This work aims to analyze a tool to aid decision making in portfolio choice in the Brazilian capital market. This tool has the foundations established by Benjamin Graham, a reference to investors since the thirties of last century. Accordingly, some of the parameters used by Graham are modeled in integer programming in VBA (Excel), whose solution is obtained using the library functions UFFLP. We proposed two modeling: first minimizing the $\mathrm{P} / \mathrm{E}$ ratio, and the second, maximizing dividends. Of portfolios resulting stands for the meaningful participation of companies in the construction, due to the low price per earnings, and energy, due to the high dividends yields. The results show that the model of Graham would not apply to valuate the chosen assets, perhaps due to simplifications in the model's employment.
\end{abstract}

Palavras-chave: Capital Market, Integer Programming, Benjamin Graham.

\footnotetext{
${ }^{1}$ Dept $^{\mathrm{o}}$ de Engenharia de Produção, Universidade Federal Fluminense. Campus Niterói. E-mail: octaviothome@gmail.com.

2 Dept $^{\mathbf{o}}$ de Engenharia de Produção, Universidade Federal Fluminense. Campus Niterói. E-mail: ribordeaux@hotmail.com.
} 


\section{Introdução}

O início do século XXI vem se caracterizando por um período importante de amadurecimento do mercado de capitais brasileiro, tendo em vista a estabilidade monetária conquistada em meados da década de 1990, o regime de metas de inflação iniciado em 1999, o crescimento da economia brasileira, dentre outros fatores, que têm proporcionado um ambiente favorável para que o investidor brasileiro busque meios diversificados para alocação de capital.

Esse cenário motiva a entrada de pequenos investidores no mercado de capitais, promovendo o aumento de liquidez dos ativos disponíveis, o incentivo a abertura de capital por parte de outras empresas e o maior fluxo de crédito a financiamentos de projetos de investimento, como na forma de debêntures e outros títulos.

Além disso, o mercado de capitais fortalecido favorece o aprimoramento dos processos de gestão por parte das empresas participantes, seja no âmbito financeiro, contábil ou ambiental. Outra questão que merece destaque é o aumento da transparência por parte das empresas na divulgação de seus resultados e de sua política de gestão.

O próprio governo brasileiro vem se beneficiando dessa nova fase de mentalidade do investidor brasileiro através da emissão de títulos para financiamento da dívida pública, os quais vêm sendo cada vez melhor avaliados pelos órgãos internacionais avaliadores de risco.

Nesse sentido, o movimento recente de inserção de novos investidores e empresas no mercado de capitais brasileiro favorece o crescimento econômico, a viabilização de projetos públicos e privados, a melhoria da infraestrutura e a geração de emprego e renda para a população.

No entanto, mesmo diante de um cenário favorável, é imprescindível que os investidores entrantes no mercado adotem uma estratégia consistente para alocação dos recursos financeiros disponíveis, no sentido de prover o acúmulo de patrimônio com rentabilidade superior aos meios mais tradicionais em se tratando de mercado brasileiro, como poupança e imóveis, e minimizando o risco do valor investido.

A literatura disponível relativa à análise de investimento e mercado de capitais oferece diversos métodos, técnicas, conceitos que objetivam propor estratégias vencedoras. Muitas destas, contemplando um complexo arcabouço matemático e computacional. Boa parte delas focando em variação instantânea de preço, a despeito da instituição, do negócio, do mercado por trás da sigla que dá nome ao ativo negociado.

Ao mesmo tempo, os fundamentos publicados pelo investidor americano Benjamin Graham há quase oito décadas e praticados por ele e seus seguidores, dentre eles Warren Buffet, têm se mostrado eficiente, mesmo em face de sua relativa simplicidade teórica e matemática. No entanto, tal simplicidade não se traduz em menor esforço por parte do investidor em avaliar as empresas com ações disponíveis no mercado.

Os fundamentos publicados adotados por Graham requerem uma minuciosa análise qualitativa das empresas listadas, o que não é abordado na elaboração da metodologia deste trabalho, mas somente na discussão dos resultados obtidos. Além disso, Graham propõe o uso de uma série de indicadores de resultados das empresas, os conhecidos múltiplos fundamentalistas, que conferem um caráter quantitativo ao seu método.

Com isso, dado que o mercado de capitais dispõe de centenas de empresas listadas, cada qual com seus respectivos indicadores de resultados e preço de mercado, o uso de uma ferramenta computacional que viabilize acelerar o processo de análise consiste em um meio de evoluir no método proposto por Graham ou outros investidores, tanto quanto na aplicação de lógicas de programação em finanças, tal como a modelagem em programação inteira, que 
tem grande aplicação em otimização em logística.

Desta forma, este trabalho objetiva avaliar a aplicação do "método Benjamin Graham" de investir no mercado de capitais brasileiro, modelando-o em Programação Inteira, lançando mão da biblioteca de funções UFFLP, desenvolvida pelo Professor Artur Alves Pessoa, da Universidade Federal Fluminense.

Para isso, na seção 2 é apresentada uma revisão bibliográfica sobre as estratégias em escolha de portfólio em mercado de capitais, assim como a aplicação de ferramentas computacionais no auxílio à tomada de decisão em investimento em ações, além de uma revisão sobre modelagem em Programação Inteira. A terceira seção apresenta uma breve revisão histórica do ambiente político, econômico e empresarial brasileiro e seus impactos sobre o mercado de capitais. A quarta seção trata dos fundamentos publicado por Benjamin Graham para investimento em ações, enquanto que a seção 5 contempla a descrição da metodologia que se pretende implementar e suas limitações. Na seção 6 são apresentados os resultados e a análise crítica dos mesmos, seguida da conclusão que consta na seção 7 do artigo.

\section{Revisão Bibliográfica}

\subsection{Estratégias em escolha de portfólio no mercado de capitais}

Inúmeras são as possibilidades de análises e métodos a serem utilizados para a escolha de uma carteira de ações. Uma das estratégias clássicas em escolha de portfólio foi proposta por Markowitz (1952), em que a teoria moderna de portfólio é descrita como um problema matemático, sendo proposto um método de análise de portfólios com base na média e na variância dos retornos, de modo que a diversificação exerce papel fundamental na minimização de riscos de mercado.
Os trabalhos de Sharpe (1964) e Lintner (1965) formularam o modelo de precificação de ativos financeiros (Capital Asset Pricing Model - CAPM). Conforme resume Ross (2011), o modelo estabelece uma relação entre o retorno esperado de um ativo e o seu beta, sendo esta letra grega a sensibilidade de um título a movimentos da carteira de mercado.

Black \& Litterman (1992) propõe uma combinação que provê uma solução intuitiva para o que, segundo ele, consistem nos maiores problemas em modelos de alocação de ativos que mais atormentam os investidores: a otimização das variâncias médias dos retornos, proposto por Markowitz (1952), e o $C A P M$ de Sharpe (1964) \& Lintner (1965).

Nesse sentido, a partir destas publicações clássicas, diversas outras abordagens em escolha e gestão de portfólio e análise de investimento são propostas na literatura, como a de José et al (2010) que compara os métodos do fluxo de caixa descontado e do Múltiplo de Ebitda para avaliação de ações.

Fortin \& Hlouskova (2011) comparam um modelo de alocação de ativos do tipo aversão à perda com modelos tradicionais de variância média e valor em risco condicional. Como resultado, os modelos de aversão à perda apresentaram melhor desempenho que os demais.

O trabalho de Hjalmarsson \& Manchev (2012) trata da análise da composição da carteira de ativos quando restrita a características diretas das ações e seus efeitos sobre a otimização da variância média do portfolio.

Cartea et al (2012) resolvem um problema de escolha de portfolio para ilustrar os benefícios de compor uma carteira de ativos com títulos indexados à inflação, utilizando dados históricos para avaliar a rentabilidade dos títulos em presença de outros ativos como ações, commodities e imóveis.

Vermeulen (2013) analisa portfolios de ativos estrangeiros em períodos de pré-crise e durante crises econômicas, de modo que o estudo 
aponta para uma correlação negativa entre participação estrangeiras em períodos de crise, enquanto que para períodos durante a crise não são observadas correlações significativas.

\subsection{Ferramentas computacionais aplicadas a investimento em ações}

O uso de softwares como ferramentas de auxílio à tomada de decisão em investimento em ações consiste em um meio eficiente de análise de dados que possibilita executar a tarefa sob um grande volume de dados, de tamanho tal que, muitas vezes torna-se humanamente inviável.

Nesse sentido, Kung (2008) faz uso de programação dinâmica estocástica para definir um modelo multi-período de alocação de ativos e deriva de uma fórmula analítica para as proporções ideais investidas em títulos de curto e longo prazo. Em seguida, o método de máxima verossimilhança é utilizado para estimar os parâmetros relevantes. Finalmente, o modelo é implementado através de algoritmo de recursão para encontrar numericamente a alocação ótima de recursos entre os títulos de curto e longo prazo para o investidor com uma concessionária de energia e um horizonte de investimento de dez anos. Os resultados mostram que o investidor tem uma proporção maior de vínculo curto, se seu horizonte de investimento fica menor e / ou se ele é mais avesso ao risco.

Xia et al (2000) propõe um modelo de seleção de carteiras em que os retornos esperados dos títulos são considerados como variáveis, e não a média dos ativos. Um algoritmo genético é proposto para resolver o problema de otimização, que é difícil de ser resolvido com os algoritmos tradicionais existentes. $\mathrm{O}$ modelo é apresentado através de um exemplo numérico e comparado com os resultados obtidos a partir do modelo tradicional de Markowitz.

Nessa mesma linha, Soleimani et al (2009) aplicam algoritmos heurísticos na solução do problema de escolha de portfolio de Markowitz. Com isso, o estudo tratou da solução de um problema de programação mista inteira não linear do tipo NP-Difícil, sendo utilizado um algoritmo genético correspondente. E assim, a etapa computacional consiste em duas fases principais: na primeira é validado o algoritmo genético proposto, e na segunda, avaliou-se a aplicabilidade do modelo em grande escala.

Mais recentemente, tomando por base os trabalhos de Markowitz (1952), Sharpe (1964) e Lintner (1965), Ustun \& Kasimbeyli (2012) propõe um modelo para escolha de portfolio. Neste trabalho, considerando o desempenho de previsão a partir de propriedades residuais, os autores formularam um modelo de variância média assimétrico com 11 funções objetivo, aplicando algoritmo FMSG.

\subsection{Modelagem em programação inteira}

Modelar um problema significa expressá-lo sob a forma de gráficos, fluxogramas, equações matemáticas ou outra forma que viabilize a sua interpretação, análise e solução. A modelagem em programação inteira consiste na tradução de um dado problema através de equações lineares, cujas variáveis e dados de entrada admitem apenas números inteiros.

Essa técnica se mostra eficiente na solução de problemas de otimização em logística, produção industrial, alocação de recursos e em finanças. Guéret (2000) ressalta que economistas muitas vezes lidam com dados incertos e naturalmente utilizam para ferramentas estatísticas, quando frequentemente um modelo matemático simples poderia resolver um problema com muitas variáveis muito rapidamente através de técnicas de otimização.

Bradley et al (1977) apresenta um exemplo de aplicação de programação inteira na tomada de decisão em investimento em projeto. Um problema típico de escolha de projeto requer a avaliação de uma série de variáveis, como custo dos insumos de cada projeto, o retorno financeiro previsto de cada um 
deles, dentre outras específicas da área em que se inserem as alternativas.

Nesse sentido, a variável binária $\boldsymbol{x}_{\boldsymbol{j}}$ indicaria se o j-ésimo projeto, dentre $n$ possíveis, seria ou não escolhido, assumindo valor 0 em caso negativo e 1 em caso positivo. Seja $\boldsymbol{c}_{j}$, o resultado esperado do $\boldsymbol{j}$-ésimo investimento e $\boldsymbol{a}_{i j}$, a quantidade de insumo $\boldsymbol{i}$ (dentre $\boldsymbol{m}$ possíveis) necessária para implantação do j-ésimo projeto, a modelagem do problema, que consiste em escolher uma composição da carteira de investimento que minimiza custo e maximiza rendimento, poderia se dar da seguinte forma:

Função Objetivo:

$$
\operatorname{Max} \sum_{j=1}^{n} x_{j} c_{j}
$$

Sujeito a:

$$
\begin{array}{ll}
\sum_{j=1}^{n} x_{j} a_{i j} \leq b_{i} & (\boldsymbol{i}=1,2, \ldots, \boldsymbol{m}) \\
x_{j}=\text { o ou } 1 & (\boldsymbol{j}=1,2, \ldots, \boldsymbol{n})
\end{array}
$$

Desta forma, para este problema tem-se apenas uma variável $\left(\boldsymbol{x}_{\boldsymbol{j}}\right)$, sendo $\boldsymbol{c}_{\boldsymbol{j}}$, $\boldsymbol{a}_{i j}$, e $\boldsymbol{b}_{\boldsymbol{i}}$ os dados de entrada, sendo que este último representa o orçamento disponível para investimento. Esta é a estrutura convencional de uma modelagem de problema a ser resolvido por programação inteira, em que inicialmente é expressa a função objetivo, e em seguida as restrições a que estão a que a função objetivo se submete. Outros exemplos de aplicação de programação inteira em finanças podem ser encontrados em Guéret (2000).

A obtenção de solução de problemas em modelos de programação inteira requer o uso de softwares que possibilitem a inserção do algoritmo que interpreta as funções objetivo e de restrição, os dados de entrada e as variáveis do problema.

Neste trabalho, os problemas propostos são resolvidos através da UFFLP, uma biblioteca de funções que possibilita a modelagem de problemas em Visual Basic for Application (VBA), que está disponível em planilhas de cálculo como o Microsoft Excel, o que facilita o tratamento dos dados utilizados pelos modelos matemáticos e a visualização dos resultados.

\section{O mercado de capitais brasileiro}

Segundo Armínio Fraga Neto, Presidente do Banco Central do Brasil no período de 1999 a 2003, o Brasil vive uma verdadeira revolução no mercado de capitais, em que pela primeira na história, empresários brasileiros têm acesso a capital de risco para investir a um custo razoável, o que pode ser atribuído aos melhores métodos de gestão praticados pelas empresas, reduzindo o risco de investimento no mercado de capitais, e a redução contínua na taxa de juros, que estimula a busca por investimentos mais rentáveis.

Mas essa trajetória foi longa. Segundo a Comissão de Valores Mobiliários (2013), antes da década de 60 , os brasileiros investiam principalmente em ativos reais (imóveis), evitando aplicações em títulos públicos ou privados. Nesse período, o desenvolvimento do mercado de capitais foi prejudicado por um ambiente de descontrole inflacionário e pela Lei da Usura (Decreto $\mathrm{n}^{\circ} 22.626$ de 07/04/1933), que limitava a taxa máxima de juros em $12 \%$ ao ano.

Segundo o Portal do Investidor, a partir da década de 60, o Governo Militar adotou medidas no âmbito econômico, resultando em diversas modificações no mercado acionário, tais como: a reformulação da legislação sobre Bolsa de Valores, a transformação dos corretores de fundos públicos em Sociedades Corretoras, forçando a sua profissionalização, a criação dos Bancos de Investimento, a quem foi atribuída a principal tarefa de desenvolver a indústria de fundos de investimento. 
A década de 1990 presenciou a aceleração do movimento de abertura da economia brasileira, resultando no aumento do volume de investidores estrangeiros atuando no mercado de capitais brasileiro. Além disso, algumas empresas brasileiras começam a acessar o mercado externo, principalmente a New York Stock Exchange, sob a forma de ADR's (American Depositary Reciepts), objetivando se capitalizar através do lançamento de valores mobiliários no exterior.

O desenvolvimento do mercado de capitais brasileiro motivou a entrada de investidores mais exigentes, acostumados a investir em mercados com práticas de governança corporativa mais avançadas que as aplicadas no mercado brasileiro, levando a um movimento contrário ao presenciado até então, quando mais empresas fecharam capital do que abriram e o volume negociado também recua.

O investimento em ações de empresas brasileiras pode ser feito através da compra de ações ordinárias e preferenciais. As primeiras conferem ao seu titular o direito de voto nas Assembleias Gerais dos acionistas, o que não é necessariamente garantido aos titulares de ações preferenciais. Estes, em geral, têm prioridade no recebimento de dividendos em relação aos titulares de ações ordinárias.

Nesse cenário, a então Bovespa (Bolsa de Valores de São Paulo) cria o Novo Mercado, tratando-se de um segmento específico de empresas que se comprometem voluntariamente a implementar as melhores práticas de Governança Corporativa. Esse movimento, associado à estabilidade econômica conquistada com a valorização internacional dos preços das commodities, a política de metas de inflação e de manutenção do superávit primário, vem proporcionando um ambiente favorável para atrair empresas e investidores.

Em função disso, é natural a inserção de novos investidores buscando proteger o próprio patrimônio da ação corrosiva da inflação e ainda obter rentabilidade sobre o capital investido superior à da poupança. Nesse sentido, esse trabalho se propõe a prover uma ferramenta consistente de auxílio à tomada de decisão na montagem de uma carteira de ações, tomando por base os fundamentos consagrados por Benjamin Graham, o que será abordado em maiores detalhes na próxima seção.

\section{Os princípios de Benjamin Graham para investimento em ações}

Arnold (2012) destaca que, para muitos, Benjamin Graham foi o maior gênio financeiro do século XX. Através de suas obras clássicas The Intelligent Investor e Security Analysis, esta última ainda sem tradução para o português, Graham descreve em detalhes seus métodos, conceitos e crenças a respeito da tarefa de compor uma carteira de investimento.

A clareza dos seus métodos, assim como a sua simplicidade e coerência, atraíram diversos seguidores do mundo das finanças, sendo o mais famoso deles o investidor Warren Buffet, gestor de uma das maiores carteiras de investimento do mundo (Hagstrom, 2005).

A noção de investimento com margem de segurança é um pilar do método adotado por Graham, que consiste em admitir a compra de um ativo que esteja subvalorizado pelo mercado, donde surge a fábula do Senhor Mercado.

$\mathrm{Em}$ The Intelligent Investor, Graham (1973) elabora a fábula do Senhor Mercado para ilustrar de forma simples em que consiste o processo de gestão de investimentos. Nesta fábula, o Senhor Mercado se dispõe a comprar seus papéis a determinados preços, ao mesmo tempo em que outros papéis são oferecidos a preços estabelecidos por Ele. Nesse sentido, cabe ao Investidor Inteligente avaliar a qualidade das oportunidades de compra e venda disponibilizadas pelo Senhor Mercado.

Para esta análise, Graham destaca a função do cálculo dos múltiplos obtidos a partir dos balanços das empresas, assim 
como a interpretação destes resultados isolados e combinados. Um dos principais indicadores utilizados é o índice preço sobre lucro, ou simplesmente $\mathrm{P} / \mathrm{L}$, que será abordado com mais detalhe nesse trabalho.

Segundo Fortuna (2011), esse índice resulta da razão do preço da ação em bolsa pelo lucro líquido anual da empresa, possibilitando estimar através dele o número de anos necessários para recuperação do investimento, desde que a empresa mantenha o mesmo lucro e o distribua integralmente.

Para Graham, um ativo a preço superior a 15 vezes o lucro médio dos últimos três anos deve ser evitado, pois está caro, e não há margem de segurança. Arnold (2012) lembra que, a margem de segurança de Graham é análoga àquelas utilizadas para construção de pontes e navios, os quais são projetados para suportar uma carga superior do que a esperada e até eventos anormais imagináveis. Daí, Graham indica que uma ação deve ser comprada não só quando está avaliada a preço mais baixo do que o calculado, mas muito mais baixo.

Além do índice P/L, Graham destaca a importância de avaliar o tamanho da empresa em que se pretende investir. Esta análise objetiva excluir companhias pequenas, mais vulneráveis a adversidades do setor em que se encontra.

A saúde financeira da empresa também é importante nessa abordagem, segundo a qual delimita que volume de os ativos circulantes seja no mínimo igual ao dobro do volume dos passivos circulantes. Da mesma forma que o endividamento de longo prazo não deve exceder o capital de giro.

A empresa avaliada deve apresentar histórico consistente de lucros. Para Graham, a empresa deve ter apresentado algum lucro em cada um dos últimos dez anos, além de crescimento mínimo de um terço nos lucros usando médias trienais no início e no fim e de pagamento ininterrupto de dividendos nos últimos vinte anos.

E ainda, o preço do ativo não deve ser superior a uma vez e meia o último valor contábil divulgado. Aqui Graham faz a seguinte ressalva: um múltiplo de lucro inferior a 15 poderia justificar um múltiplo de ativos maior. Com isso, sugere-se que o produto desses múltiplos não seja superior a 22,5 , que é o resultado do produto de 15 por 1,5 .

Desta forma, tomando por base, o que passamos a denominar, as restrições de Graham, é possível a modelagem computacional dessas restrições de modo a prover um algoritmo que automatize esse processo de análise, pelo menos essa etapa da análise, o que pode ser executado através de programação inteira, que é o tema da próxima seção.

\section{Metodologia}

\subsection{Descrição do método}

Para implementação da estratégia proposta, faz-se necessário primeiramente delimitar que grupo de ações será submetido ao modelo. Neste trabalho, as ações do índice IBrX (Índice Brasil) e seus indicadores fundamentalistas serão os dados de entrada da modelagem.

Conforme informado no sítio da BM\&FBovespa, o IBrX é um índice de preços que mede o retorno de uma carteira teórica composta por 100 ações selecionadas entre as mais negociadas na Bovespa, em termos de número de negócios e volume financeiro, conforme Tabela 01.

Essas ações são ponderadas na carteira do índice pelo seu respectivo número de ações disponíveis à negociação no mercado. 
Tabela 01: Composição do IBrX em 08/03/2013

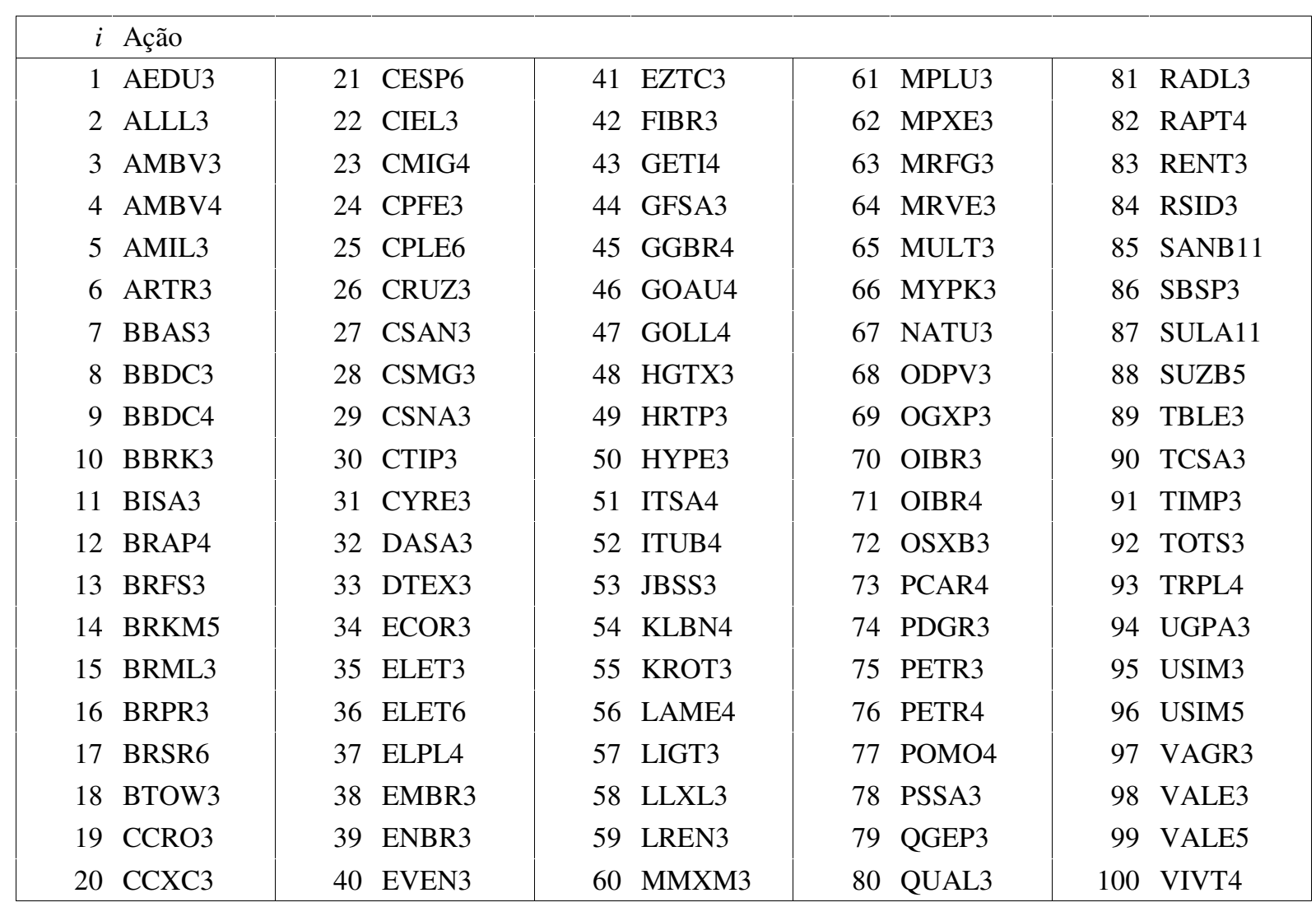

Fonte: BM\&FBovespa

Antes da implementação da metodologia proposta, cabe a ressalva de que algumas empresas possuem dois tipos de ações listadas no IBrX, ordinárias e preferenciais. Em função disso, a fim de garantir a diversificação da carteira resultante e dada a preferência explicitada por Graham pelas ações ordinárias, serão excluídas da base de dados as respectivas ações preferenciais, o que resulta em 93 ativos disponíveis.

Assim, os 93 ativos são listados em planilha e os seguintes dados referentes a cada ativo são registrados: ativos circulantes; passivos circulantes; capital de giro; dividendos; dívida de longo prazo; preço; e índice preço sobre lucro, obtidos na página da internet "FUNDAMENTUS". Outro dado de entrada importante é o capital disponível para investimento.

Conforme será apresentado a seguir, uma das carteiras será escolhida minimizando o índice P/L do portfólio. Nesse sentido, face às ressalvas feitas por Graham quanto às empresas que apresentam P/L negativo, para estas os respectivos índices serão alterados para um número elevado (igual a 999.999), de modo que a modelagem descarte tais ativos como opção para escolha.

A partir daí, duas estratégias são propostas: a primeira objetivando minimizar o índice P/L da carteira; e a segunda, maximizar dividendos, conforme a modelagem a seguir. Como as duas propostas são submetidas às restrições do método Graham de investir, o que diferencia as carteiras são as funções objetivo, conforme a seguir. 


\section{Dados de Entrada:}

$i \equiv \operatorname{ativo}(i=1, \ldots, 93)$

$a c_{i} \equiv$ ativo circulante da empresa $i[\mathrm{R} \$$ ]

$p c_{i} \equiv$ passivo circulante da empresa $i[\mathrm{R} \$]$

$c g_{i} \equiv$ capital de giro da empresa $i[\mathrm{R} \$], c g_{i}=a c_{i}-p c_{i}$

$d y_{i} \equiv$ dividendos distribuídos aos acionistas $i$ [ \% sobre capital investido ]

$d v_{i} \equiv$ dívida de longo prazo da empresa $i[\mathrm{R} \$$ ]

$p_{i} \equiv$ preço da ação $i[\mathrm{R} \$]$

$p l_{i} \equiv$ índice preço sobre lucro da ação $i$ [ adimensional ]

$c d \equiv$ capital disponível para investimento [ $\mathrm{R} \$$ ] $(\mathrm{R} \$ 100.000,00)$

\section{Variáveis:}

$x_{i} \equiv$ quantidade a ser adquirida da ação $i$ [ lotes de 100 ], $x_{i}=1, \ldots, 50$

$\mathrm{y} \equiv$ índice preço sobre lucro do portfólio resultante, de modo que: $y=\frac{p l_{i}}{c d} x_{i}$

$\mathrm{z} \equiv$ dividendo do portfólio resultante, de modo que: $z=\frac{d y_{i}}{\sigma d} x_{i}$

\section{Função Objetivo:}

$1^{\mathrm{a}}$ Carteira (minimiza $P / L$ )

Miny $2^{\mathrm{a}}$ Carteira (maximiza dividendo)

$\operatorname{Maxz}$

Sujeito a:

$$
\begin{aligned}
& \sum_{i=1}^{93} p_{i} x_{i} \leq c d \\
& p_{i} x_{i} \leq \frac{c d}{4} \\
& \sum_{i=1}^{93}\left(\frac{p_{i}}{c d}\right) p l_{i} x_{i} \leq 15 \\
& \sum_{i=1}^{93}\left(\frac{p_{i}}{c d}\right)\left(\frac{a c_{i}}{p c_{i}}\right) x_{i} \geq 2 \\
& \sum_{i=1}^{93}\left(\frac{p_{i}}{c d}\right)\left(c g_{i}-d v_{i}\right) x_{i} \geq 0
\end{aligned}
$$

\subsection{Limitações do método}

Conforme diversos outros trabalhos disponíveis na literatura acadêmica (vide os apresentados na seção 2.2 deste artigo), este estudo se propõe a apresentar a aplicabilidade de uma ferramenta computacional no auxílio à tomada de decisão em investimento em ações, de modo a compor a modelagem com as restrições consagradas pelo investidor Benjamin Graham.

No entanto, cabe a ressalva de que a filosofia proposta por Graham não contempla somente informações de natureza quantitativa, como os múltiplos 
utilizados neste trabalho. Em The Intelligent Investor (1973), Graham lista quesitos de caráter qualitativo que devem ser avaliados pelo investidor no processo de tomada de decisão, chamando atenção inclusive para as notas de rodapé dos relatórios financeiros anuais das empresas listadas.

Nesse sentido, esse trabalho não se propõe a modelar toda "Filosofia Benjamin Graham" para investimento em ações através da programação. Mas somente, se utilizar dos indicadores mensuráveis utilizados por ele para estabelecer a série de restrições a serem impostas sobre a carteira a ser escolhida.

\section{Resultados e Discussão}

Da modelagem proposta, foram obtidas duas carteiras com as composições apresentadas nas figuras $1 \mathrm{e}$ 2. Aqui, cabe atentar para o fato de que a modelagem proposta não impõe que seja utilizado um percentual mínimo do capital disponível, e como a função objetivo minimiza o índice preço sobre lucro da carteira resultante, o que resultaria em um portfólio isento de ativos com $\mathrm{P} / \mathrm{L}$ igual a zero. Para corrigir tal distorção, foi adicionada a restrição de que o capital investido deveria ser superior a $95 \%$ do capital disponível.

$\mathrm{O}$ índice preço sobre lucro da carteira é de 8,043. Outra questão que merece destaque é a forma pela qual o capital disponível foi distribuído. Isto porque, $85 \%$ do capital utilizado foram alocados em apenas três empresas: Bradespar (Holding Diversificada); Transmissão Paulista (Energia Elétrica), Even (Construção Civil) e MRV (Construção Civil). Os $15 \%$ restantes alocados em Itaú Unibanco (Instituição Financeira), Rossi Engenharia e EZTec (Construção Civil), AES Tiete (Energia Elétrica) e BR Brokers (Intermediação Imobiliária).

\begin{tabular}{|lrcr|}
\hline \multicolumn{4}{|c|}{ Composição da Carteira IBrX Minimiza P/L } \\
\hline \multicolumn{1}{|c}{ Ação } & Quantidade & Valor & Proporção \\
\hline BRAP4 & 900 & $\mathrm{R} \$ 23.769,00$ & $24,92 \%$ \\
TRPL4 & 600 & $\mathrm{R} \$ 19.764,00$ & $20,72 \%$ \\
EVEN3 & 2000 & $\mathrm{R} \$ 18.940,00$ & $19,85 \%$ \\
MRVE3 & 1900 & $\mathrm{R} \$ 18.829,00$ & $19,74 \%$ \\
ITUB4 & 200 & $\mathrm{R} \$ 7.192,00$ & $7,54 \%$ \\
EZTC3 & 100 & $\mathrm{R} \$ 2.604,00$ & $2,73 \%$ \\
GET14 & 100 & $\mathrm{R} \$ 1.941,00$ & $2,03 \%$ \\
RSID3 & 500 & $\mathrm{R} \$ 1.640,00$ & $1,72 \%$ \\
BBRK3 & 100 & $\mathrm{R} \$ 714,00$ & $0,75 \%$ \\
& Capital utilizado $=$ & $\mathrm{R} \$ 95.393,00$ & \\
\hline
\end{tabular}

Figura 1: Carteira obtida com $\mathrm{P} / \mathrm{L}$ mínimo

Com isso, obteve-se uma carteira com $44 \%$ no setor de Construção e $23 \%$ em Energia Elétrica. Esse resultado pode ser atribuído ao forte crescimento da indústria de construção civil na última década impulsionado principalmente pelas políticas de crescimento de crédito imobiliário, rendendo lucros significativos para o setor. No entanto, a estabilização dos preços dos imóveis somada aos problemas enfrentados por algumas construtoras na entrega de empreendimentos motivou a desconfiança do mercado. Com isso, tais empresas vêm tendendo a apresentar baixos índices P/L.

Da carteira com foco em dividendos, foi obtido um portfolio com pagamento de $15,2 \%$ de dividendo sobre o capital investido. Desta carteira percebe-se que mesmo empresas que pagam baixos dividendos foram elencadas, tais como Even e Itaú Unibanco. A primeira compensa com baixo $\mathrm{P} / \mathrm{L}$ e a segunda com elevada diferença positiva entre capital de giro e dívida. A empresa que mais contribui para o dividendo e á Eletropaulo, com $32,8 \%$. 


\begin{tabular}{|lrcr|}
\hline \multicolumn{4}{|c|}{ Composição da Carteira IBrX Maximiza Dividendo } \\
\hline \multicolumn{1}{c}{ Ação } & Quantidade & \multicolumn{1}{c|}{ Valor } & Proporção \\
\hline ELPL4 & 2200 & $\mathrm{R} \$ 24.970,00$ & $25,11 \%$ \\
GET14 & 1200 & $\mathrm{R} \$ 23.292,00$ & $23,42 \%$ \\
ITUB4 & 600 & $\mathrm{R} \$ 21.576,00$ & $21,70 \%$ \\
RSID3 & 4600 & $\mathrm{R} \$ 15.088,00$ & $15,17 \%$ \\
ARTR3 & 200 & $\mathrm{R} \$ 4.250,00$ & $4,27 \%$ \\
BRAP4 & 100 & $\mathrm{R} \$ 2.641,00$ & $2,66 \%$ \\
BBRK3 & 300 & $\mathrm{R} \$ 2.142,00$ & $2,15 \%$ \\
MRVE3 & 200 & $\mathrm{R} \$ 1.982,00$ & $1,99 \%$ \\
CYRE3 & 100 & $\mathrm{R} \$ 1.646,00$ & $1,66 \%$ \\
EVEN3 & 100 & $\mathrm{R} \$ 947,00$ & $0,95 \%$ \\
ODPV3 & 100 & $\mathrm{R} \$ 914,00$ & $0,92 \%$ \\
& Capital utilizado & $\mathrm{R} \$ 99.448,00$ & \\
\hline
\end{tabular}

Figura 2: Carteira obtida com dividendo máximo

Destes resultados tem-se uma expressiva participação das empresas dos setores de construção civil e energia elétrica. As primeiras oferecem baixo $\mathrm{P} / \mathrm{L}$, enquanto as últimas, bons dividendos.

Dos resultados obtidos, foi possível verificar que determinados ativos foram escolhidos para compor ambas as carteiras. Isso pode ser explicado pelo fato de que as restrições de Graham foram aplicadas ao portfolio, e não a cada ativo individualmente.

Consequentemente, ativos com bons indicadores a atender às restrições de Graham serviram de "lastro" para a inserção de ativos com as características desejadas pela função objetivo, que no primeiro caso foi o baixo índice preço sobre lucro, e no segundo o elevado pagamento de dividendos.

No entanto, o termo " $\left(p_{i} / c d\right) * x_{i}$ ", presente nas equações 08,09 e 10 , pondera as características de cada empresa conforme a participação dos respectivos ativos na composição da carteira. Essa estratégia limita a participação de empresas com dados econômico-financeiros significativamente distantes daqueles utilizados por Graham.

Nesse sentido, cabe destacar que, possivelmente, caso a filosofia utilizada por Benjamin Graham fosse aplicada na íntegra para os ativos avaliados neste trabalho, nenhum deles seria escolhido pelo investidor. Cabe reforçar que este trabalho não objetivou modelar os critérios de Graham na sua integralidade, o que nesse caso deveria contemplar inclusive as análises das notas de rodapé dos Relatórios de Resultados das empresas, como a gestão dos derivativos financeiros.

Por outro lado existe o fato de que os resultados obtidos se devem a dados econômico-financeiros passados, submetidos à conjuntura econômica da época, não representando garantia de resultados futuros. Tal característica, além de convergir com o método de Graham, está em consonância com a proposta deste trabalho, que não objetivou prever o comportamento futuro dos preços dos ativos, mas analisar uma estratégia de auxílio à tomada de decisão em investimento no mercado de capitais brasileiro.

\section{Conclusão}

$\mathrm{O}$ presente trabalho objetivou analisar uma proposta de estratégia de alocação de capital no mercado de capitais brasileiro aplicando os conceitos consagrados por Benjamin Graham e modelagem em programação inteira, utilizando como ferramenta a biblioteca de funções UFFLP.

Sua principal motivação reside no fato de que o momento histórico para investimento no mercado de capitais brasileiro é favorável, o que pôde ser evidenciado através da revisão histórico do cenário político, econômico e empresarial brasileiro entre as décadas de 1960 e 1990, conforme apresentado na seção 3 .

Com isso, da mesma forma como diversos outros trabalhos disponíveis na literatura acadêmica, este tem como contribuição analisar uma ferramenta computacional de auxílio à tomada de decisão em investimento no mercado de capitais, de modo a viabilizar uma maior rapidez na análise de dezenas de ativos disponíveis.

Conforme expressado em sua obra mais conhecida, The Intelligent Investor (1973), Graham se vale tanto de análises quantitativas das empresas através dos indicadores econômico-financeiros apresentados neste trabalho, quanto de análises qualitativas através da avaliação 
do negócio como um todo, considerando seus concorrentes, valor da marca, dentre outros fatores. Consequentemente, a modelagem integral dos fundamentos de Graham se mostra inviável, o que não impossibilitou o uso de seus fundamentos quantitativos na elaboração de uma ferramenta de auxílio à tomada de decisão em investimento em ações, conforme apresentado neste trabalho.

É possível depreender deste trabalho que outras modelagens são possíveis, segundo a estratégia do investidor, que pode restringir seu portfólio segundo os métodos de outros grandes investidores, como Phillip Fisher, Warren Buffet, Peter Lynch e outros. Além de mudar a função objetivo, focando em lucro, ativos e dívida, por exemplo.

Desta forma, diversas outras abordagens e melhorias são possíveis a partir deste trabalho, seja pela modelagem em programação inteira de outras estratégias ou da modelagem dos fundamentos de Benjamin Graham em outros algoritmos ou métodos de programação. Seria possível ainda, propor outras funções objetivos, que não as então apresentadas, como a maximização de ativos líquidos, minimização de dívidas de curto prazo, dentre outras.

\section{Referências}

ARNOLD, G. (2012). Os Grandes Investidores. Editora Saraiva, São Paulo.

BANCO CENTRAL DO BRASIL (2011). Dez anos de metas para a inflação no Brasil: 1999-2009, Brasília.

BLACK, F. \& LITTERMAN, R. (1992). "Global Portfolio Optimization". Financial Analysts Journal, vol. 48, p. 2843.

BM\&FBOVESPA, <http://www.bmfbov espa.com.br/indices/ResumoIndice.aspx? Indice $=\mathrm{IBrX} \& \mathrm{Idi}$ oma $=\mathrm{pt}-\mathrm{br}>$, acesso em 08/03/2013.
BRADLEY, S. P., HAX, A. C. \& MAGNANTI, T. L. (1977). Applied Mathematical Programming. AddisonWesley.

BRASIL (1933). Decreto $\mathrm{n}^{\circ} 22.626$ de 07/04/1933, disponível em https://www .planalto.gov.br/ccivil_03/decreto/d22626 .htm, acesso em 28/05/2013.

CARTEA, A., SAÚL, J. \& TORO, J. (2012). Optimal portfolio choice in real terms: Measuring the benefits of TIPS. Journal of Empirical Finance, vol. 19, p. 721-740.

COMISSÃO DE VALORES MOBILIÁRIOS (2013). Mercado de Valores Mobiliários Brasileiro, Rio de Janeiro.

FORTIN, I. \& HLOUSKOVA, J. (2011). Optimal asset allocation under linear loss aversion. Journal of Banking and Finance, vol. 35, p. 2974-2990.

FORTUNA, E. (2011). Mercado Financeiro - Produtos e Serviços. Editora QualityMark, $18^{a}$ Edição, São Paulo.

FUNDAMENTUS, <http://www.fundam entus.com.br/>, acesso em 10/03/2013.

GAPSO - TECNOLOGIA DE DECISÃO, http://www.gapso.com.br/uff lp/, acesso em 08/03/2013.

GRAHAM, B. (1973). The Intelligent Investor. Perfect Bound, $4^{\text {th }}$ Edition, New York.

GUÉRET, C., PRINS, C. \& SEVAUX, M. (2000). Application of optimization with Xpress-MP. Eyrolles, Paris.

HAGSTROM, R. G. (2005). The Warren Buffet Way. John Wiley \& Sons, Second Edition, New Jersey.

HJALMARSSON, E. \& MANCHEV, P. (2012). Characteristic-based meanvariance portfolio choice. Journal of Banking and Finance, vol. 36, p. 13921401. 
JOSÉ, B. O. S, JUNIOR, W. L. S. \& BORDEAUX-RÊGO, R. (2010) Análise de metodologias para avaliação de empresas: estudo aplicado ao caso da América Latina Logística. ENGEVISTA, vol. 12, n. 2, p. 93-107.

KUNG, J. J. (2008). Multi-period asset allocation by stochastic dynamic programming. Applied Mathematics and Computation, vol. 199, p. 341-348.

LINTNER, J. (1965). The valuation of risk assets and the selection of risky investments in stock portfolios and capital budgets. The Review of Economics and Statistics, vol. 47, p. 1337.

MARKOWITZ, H. (1952). Portfolio Selection. The Journal of Finance, vol. 7, p. 77-91.

PORTAL DO INVESTIDOR, $<$ http://www.portaldoinvestidor.gov.br/m enu/Menu_Academico/O_Mercado_de_v alores_mobiliarios_brasileiro/Historia_M ercado-Capitais.html>, acesso em 07/03/2013.

ROSS, S. A., WESTERFIELD, R. W. \& JAFFE, J. F. (2011). Administração Financeira - Corporate Finance. Atlas, $2^{\mathrm{a}}$ edição, São Paulo.

SHARPE, W. F. (1964). Capital Asset Prices: A theory of market equilibrium under conditions of risk. The Journal of Finance, vol. 19, p. 425-442.

SOLEIMANI, H., GOLMAKANI, H. R., SALIMI, M. H. (2009). Markowitz-based portfolio selection with minimum transaction lots, cardinality constraints and regarding sector capitalization using genetic algorithm. Expert Systems with Applications, vol. 36, p. 5058-5063.

USTUN, O. \& KASIMBEYLI, R. (2012). Combined forecasts in portfolio optimization: A generalized approach. Computers \& Operations Research, vol. 39 , p. 805-819.
VERMEULEN, R. (2013). International diversification during the financial crisis: A blessing for equity investors? Journal of International Money and Finance, vol. 35, p. 104-123.

XIA, Y., LIU, B., WANG, S. \& LAI, K. K. (2000). A model for portfolio selection with order of expected returns. Computers \& Operations Research, vol. 27 , p. 409-422. 